\title{
High-Speed Analysis of Heating and Current Drive with Neutral Beam Injection in Tokamak Plasma*)
}

\author{
Ryoya FUNABASHI, Takaaki FUJITA and Atsushi OKAMOTO \\ Graduate School of Engineering, Nagoya University, Furo-cho, Chikusa-ku, Nagoya, Aichi 464-8603, Japan
}

(Received 5 January 2020 / Accepted 16 August 2020)

\begin{abstract}
We developed a Fokker-Planck solver to analyze heating and current drive with neutral beam injection in tokamak plasma and introduced it into the integral transport code TOTAL. In the developed solver, the fast ion distribution function is expanded in the eigenfunctions of the collisional pitch angle scattering operator. The time evolution of the distribution function is obtained by solving 1-D partial differential equations for velocity, so that high-speed analysis is possible. In the analyses using the point source and assuming zero toroidal drift, the results of the heating power and the driven current evaluated in steady state agreed well with the results calculated by the OFMC code and the ACCOME code. We demonstrated that the TOTAL code implemented with the solver is able to simulate a DEMO operation scenario with neutral-beam injection.
\end{abstract}

(C) 2020 The Japan Society of Plasma Science and Nuclear Fusion Research

Keywords: TOTAL, neutral beam injection, Fokker-Planck solver, simulation, current drive, heating

DOI: $10.1585 /$ prr.15.2401071

\section{Introduction}

In Japan, DEMO designs are progressing to realize fusion reactors by $2050 \mathrm{~s}$. In development of the design it is needed to determine actuator specifications, so that we have to consider many operation scenarios (e.g. keeping rating fusion power and keeping full non-inductive current drive) using various actuator parameters (e.g. maximum supply fuel speed and maximum neutral beam power) with a low-computational-cost integrated transport code. The TOTAL code [1] is a 1-D transport and 2-D equilibrium calculation code, where the various physical processes (e.g. sawtooth oscillation) are described by simple models. The TOTAL code is thus suitable for the lowcomputational-cost simulation. In addition, the TOTAL code is able to simulate operation keeping rating fusion power with feed-back control of fueling rate. However, the TOTAL code was only possible to give fixed radial profiles for heating power and driven current. So, a lowcomputational-cost solver is needed to analyze the time evolution of the external heating and current drive with actuators.

In this study, we developed a low-computational-cost Fokker-Planck solver (FP solver) for the distribution functions of fast ions generated by Neutral Beam (NB) injection and introduced the developed solver into the TOTAL code to analyze the NB heating and current drive. Among the codes to solve Fokker Planck equation developed so far, there are the BAFP code [2] in the TOPICS [3] code and RISK [4]. In the BAFP code the Fokker Planck equation is solved as the 2-D partial differential equation of

author'se-mail: fujita@energy.nagoya-u.ac.jp

*) This article is based on the presentation at the 28th International Toki Conference on Plasma and Fusion Research (ITC28). the velocity and pitch angle parameters. In the developed solver, the fast ion distribution function is expanded in the eigenfunctions of the pitch angle operator in order to reduce the calculation time because the eigenfunctions are decided by the MHD equilibrium only and the time development is described by 1-D partial differential equations for the velocity. In RISK, the eigenfunctions are calculated assuming circular cross-sections of flux surfaces; in contrast we calculate the eigenfunctions for accurate shapes of flux surfaces. The developed solver is based on that in the ACCOME code [5], which analyzes NB current drive in steady state. The distribution function of fast ions in tokamak is analyzed by solving the bounce averaged FokkerPlanck equation on a magnetic surface using the finite difference method for velocity and eigenfunctions expansion in the pitch angle parameter.

In this article, first, we describe the bounce averaged Fokker-Planck equation used in the solver in section 2. Secondly, we describe the equations to evaluate NB-driven current and heating power to bulk ions and electrons from the distribution function in section 3. Next, we show the verification of the solver on driven current and heating power with an orbit following code OFMC [6] and the ACCOME code in section 4. In section 5, we describe the flow chart of the TOTAL code implemented with the developed solver and show a simulation of DEMO operation scenario with neutral-beam injection. Finally, we conclude this study in Section 6.

\section{Bounce Averaged Fokker-Planck Equation}

In order to derive the bounce averaged Fokker-Planck 
equation for fast ions generated with NB injection in a tokamak plasma, we start from the gyro-averaged FokkerPlanck equation [7]

$$
\frac{\partial f}{\partial t}+v_{\mathrm{g}} \cdot \nabla f=\sum_{\mathrm{j}} C_{\mathrm{bj}}(f)+S-L(f),
$$

where $f$ is the distribution function, $f=f(t, v, \mu, \psi, \theta)$; here $v$ is the fast ion velocity, $\mu$ is the magnetic moment $\mu=\left(m v_{\perp}^{2}\right) / 2 B, v_{\perp}$ is the velocity component perpendicular to the magnetic field, $B$ is the magnitude of magnetic field at poloidal position $\theta$ on the magnetic surface labeled by the poloidal flux function $\psi ; v_{\mathrm{g}}$ is the guiding center velocity; $C_{\mathrm{bi}}(f)$ is the collision operator between particle species $\mathrm{j}$ and fast ions; $S$ is the fast ion source term caused by ionization of injected NB particles; $L$ is the loss term. In this study, we do not consider the loss of fast ions including orbit loss and charge-exchange loss during slowing-down and then do not employ the loss term; the thermalized ions are removed through the lower boundary of the velocity space.

Here, we define the local pitch angle parameter $\eta$ as

$$
\eta^{2}=\frac{v_{\|}^{2}}{v^{2}}=1-\frac{2 B}{m v^{2}} \mu=1-\frac{\left(1-\xi^{2}\right) B}{B_{\min }},
$$

where, $v_{\|}$is the parallel velocity component and $B_{\min }$ is the minimum magnitude of field on the magnetic surface. $\xi$ is the value of $\eta$ at a point where $B=B_{\min }$ on the magnetic surface. So, we can use $\xi$ as an independent variable instead of $\mu ; f=f(t, v, \psi, \xi, \theta)$.

Now, we make two assumptions for simplifying the equation as done in [5]: we assume (i) that fast ions are in the banana regime and (ii) that the toroidal drift of fast ions can be neglected so that fast ions follow the magnetic field line. Assumption (ii) means $\boldsymbol{v}_{\mathrm{g}}=\boldsymbol{v}_{\|}$. Assumption (i) means that each ion can complete many nearly identical orbits in the poloidal plane before its orbit is significantly perturbed by collisions. In result of assumptions (i) and (ii), we can neglect $\theta$ dependence of $f$ namely $f=f_{0}(t, v, \psi, \xi)$, where $f_{0}$ means the zeroth order distribution function in $\tau_{\mathrm{B}} / \tau_{\mathrm{C}}$; $\tau_{\mathrm{B}}$ means the bounce time and $\tau_{\mathrm{C}}$ means the collision time. Namely $f$ is independent of the poloidal position in the orbit. So, we neglect the term about $\boldsymbol{v}_{\mathrm{g}}$ of eq. (1).

The bounce time $\tau_{\mathrm{B}}$ that each fast ion completes its orbit in the poloidal plane is definedas

$$
\tau_{\mathrm{B}}=\oint \frac{d l}{v_{\|}}=\oint \frac{d l_{\mathrm{p}}}{v_{\mathrm{p}}}=\oint \frac{B d l_{\mathrm{p}}}{v_{\|} B_{\mathrm{p}}}=\frac{1}{v} \oint \frac{B d l_{\mathrm{p}}}{\eta B_{\mathrm{p}}} .
$$

Here $d l$ is the line element along the field line, $d l_{\mathrm{p}}$ is the line element along the poloidal direction on the magnetic surface, $v_{\mathrm{p}}$ is the velocity of the guiding center along $d l_{\mathrm{p}}$, and $B_{\mathrm{p}}$ is the magnitude of the poloidal field. The contour integral $\oint$ is taken for a path completing the particle orbit in the poloidal direction in this article. We define a bounceaverage operator as

$$
\langle X\rangle_{\mathrm{B}}=\oint \frac{X d l}{\tau_{\mathrm{B}} v_{\|}}=\oint \frac{X d l_{\mathrm{p}}}{\tau_{\mathrm{B}} v_{\mathrm{p}}}
$$

$$
=\oint \frac{X B d l_{\mathrm{p}}}{\tau_{\mathrm{B}} v_{\|} B_{\mathrm{p}}}=\frac{1}{v \tau_{\mathrm{B}}} \oint \frac{X B d l_{\mathrm{p}}}{\eta B_{\mathrm{p}}} .
$$

We use the bounce-average operator for the gyro-averaged Fokker-Planck equation for $f_{0}$, then

$$
\frac{\partial f_{0}}{\partial t}=\sum_{\mathrm{j}}\left\langle C_{\mathrm{bj}}\left(f_{0}\right)\right\rangle_{\mathrm{B}}+\langle S\rangle_{\mathrm{B}}
$$

Now, we assume that fast ions collide only with isotropic Maxwellian bulk electrons and ions. Then $C_{\mathrm{bj}}$ is given in the following form [8]

$$
\begin{aligned}
C_{\mathrm{bj}}= & D_{\eta, \mathrm{j}}(v) \frac{\partial}{\partial \eta}\left[\left(1-\eta^{2}\right) \frac{\partial f_{0}}{\partial \eta}\right]+\frac{1}{v^{2}} \frac{\partial}{\partial v}\left(A_{v, \mathrm{j}}(v) f_{0}\right) \\
& +\frac{1}{v^{2}} \frac{\partial}{\partial v}\left(v^{2} B_{v, \mathrm{j}}(v) \frac{\partial f_{0}}{\partial v}\right),
\end{aligned}
$$

where

$$
\begin{aligned}
A_{v, \mathrm{j}} & =\frac{1}{\tau_{\mathrm{s}}} \frac{3 \sqrt{\pi}}{4} \frac{m_{\mathrm{e}}}{m_{\mathrm{b}}} v_{\mathrm{th}, \mathrm{e}}^{3}\left(\frac{m_{\mathrm{b}}}{m_{\mathrm{j}}} \frac{\ln \Lambda_{\mathrm{j}} Z_{\mathrm{j}}^{2} n_{\mathrm{j}}}{\ln \Lambda_{\mathrm{e}} n_{\mathrm{e}}} G\left(\frac{v}{v_{\mathrm{th}, \mathrm{j}}}\right)\right), \\
B_{v, \mathrm{j}} & =\frac{1}{\tau_{\mathrm{s}}} \frac{3 \sqrt{\pi}}{4} \frac{m_{\mathrm{e}}}{m_{\mathrm{b}}^{2}} \frac{T_{\mathrm{j}}}{v^{3}} v_{\mathrm{th}, \mathrm{e}}^{3}\left(\frac{m_{\mathrm{b}}}{m_{\mathrm{j}}} \frac{\ln \Lambda_{\mathrm{j}} Z_{\mathrm{j}}^{2} n_{\mathrm{j}}}{\ln \Lambda_{\mathrm{e}} n_{\mathrm{e}}} G\left(\frac{v}{v_{\mathrm{th}, \mathrm{j}}}\right)\right),
\end{aligned}
$$

and

$$
D_{\eta, \mathrm{j}}=\frac{1}{\tau_{\mathrm{S}}} \frac{3 \sqrt{\pi}}{8} \frac{m_{\mathrm{e}}}{m_{\mathrm{b}}^{2}} \frac{T_{\mathrm{j}}}{v^{5}} v_{\mathrm{th}, \mathrm{e}}^{3}\left(\frac{m_{\mathrm{b}}}{m_{\mathrm{j}}} \frac{\ln \Lambda_{\mathrm{j}} Z_{\mathrm{j}}^{2} n_{\mathrm{j}}}{\ln \Lambda_{\mathrm{e}} n_{\mathrm{e}}} F\left(\frac{v}{v_{\text {th, }}}\right)\right) .
$$

Here, $n$ is the density, $T$ is the temperature, $m$ is the mass, $Z$ is the charge number and $\ln \Lambda$ is the Coulomb logarithm for collisions between fast ions and bulk particles, subscript $j$ denotes particle species $(\mathrm{j}=\mathrm{e}$ for bulk electron, $\mathrm{i}$ for bulk ion), and subscript b means fast ion. $A_{v, \mathrm{j}}, B_{v, \mathrm{j}}$ and $D_{\eta, \mathrm{j}}$ are the friction, diffusion and scattering coefficients. The thermal velocity $v_{\text {th }}$, the critical velocity $v_{\mathrm{c}}$ the slowing down time $\tau_{\mathrm{s}}$, the function $G(x)$ and the function $F(x)$ are given in the following forms:

$$
\begin{aligned}
& v_{\mathrm{th}, \mathrm{j}}=\sqrt{\frac{2 T_{\mathrm{j}}}{m_{\mathrm{j}}}}, \\
& \tau_{\mathrm{s}}=\frac{4 \pi \varepsilon_{0}^{2} m_{\mathrm{b}}^{2} v_{\mathrm{c}}^{3}}{e^{4} Z_{\mathrm{b}}^{2} Z_{1} n_{\mathrm{e}} \ln \Lambda_{\mathrm{e}}} \approx \frac{m_{\mathrm{b}}}{m_{\mathrm{p}}} \frac{2\left(T_{\mathrm{e}}[\mathrm{keV}]\right)^{1.5}}{\ln \Lambda_{\mathrm{e}} Z_{\mathrm{b}}^{2} n_{\mathrm{e}}\left[10^{19} \mathrm{~m}^{-3}\right]}, \\
& v_{\mathrm{c}}=\left(\frac{3 \sqrt{\pi}}{4} \frac{m_{\mathrm{e}} Z_{1}}{m_{\mathrm{b}}}\right)^{1 / 3} v_{\mathrm{th}, \mathrm{e}}, \\
& Z_{1}=\sum_{j=i_{1}, i_{2}, \ldots} \frac{m_{\mathrm{b}}}{m_{\mathrm{j}}} \frac{\ln \Lambda_{\mathrm{j}} Z_{\mathrm{j}}^{2} n_{\mathrm{j}}}{\ln \Lambda_{\mathrm{j}} n_{\mathrm{e}}}=\frac{4}{3 \sqrt{\pi}} \frac{m_{\mathrm{b}} v_{\mathrm{c}}^{3}}{m_{\mathrm{e}} v_{\mathrm{th}, \mathrm{e}}^{3}}, \\
& F(x)=\operatorname{erf}(x)-x \frac{\partial \operatorname{erf}(x)}{\partial x}, \\
& F\left(2 x^{2}-1\right) \operatorname{erf}(x)+x \frac{\partial \operatorname{erf}(x)}{\partial x},
\end{aligned}
$$

where $m_{\mathrm{p}}$ is the proton mass, $\varepsilon_{0}$ is the dielectric constant of free space and $\operatorname{erf}(x)$ is the error function. If we use an approximate of $v_{\text {th,i }} \ll v \ll v_{\text {th,e }}$, we can describe eq. (7) as following forms: 
for electrons $(j=e)$,

$$
\begin{aligned}
& A_{v, \mathrm{e}}=\frac{v^{3}}{\tau_{\mathrm{s}}}, \\
& B_{v, \mathrm{e}}=\frac{T_{\mathrm{e}}}{m_{\mathrm{b}} \tau_{\mathrm{s}}},
\end{aligned}
$$

and

$$
D_{\eta, \mathrm{e}}=0
$$

for ions $(j=i 1, i 2, \ldots)$,

$$
\begin{aligned}
& \sum_{\mathrm{j}=\mathrm{i}_{1}, \mathrm{i}_{2}, \ldots} A_{v, \mathrm{j}}=\frac{v_{\mathrm{c}}^{3}}{\tau_{\mathrm{s}}}, \\
& \sum_{\mathrm{j}=\mathrm{i}_{1}, \mathrm{i}_{2}, \ldots} B_{v, \mathrm{j}}=\frac{T_{\mathrm{i}} v_{\mathrm{c}}^{3}}{m_{\mathrm{b}} \tau_{\mathrm{s}} v^{3}},
\end{aligned}
$$

and

$$
\sum_{\mathrm{j}=\mathrm{i}_{1}, \mathrm{i}_{2}, \ldots} D_{\eta, \mathrm{j}}=\frac{v_{\mathrm{c}}^{3}}{2 v^{3} \tau_{\mathrm{s}}} \frac{Z_{\mathrm{eff}}}{Z_{1}}
$$

Note that in a low velocity range, $v \sim v_{\text {th,i }}$, the assumption $v_{\text {th,i }} \ll v \ll v_{\text {th,e }}$ is not satisfied and then the distribution function obtained using the coefficients shown above would be inaccurate. However, this would have only small effects on the fast ion current since the contribution of low velocity ions to fast ion current is small.

In eq. (5), the bounce-averaged collision operator is given in following form by using eq. (2);

$$
\begin{aligned}
\left\langle C_{\mathrm{bj}}\right\rangle_{\mathrm{B}}= & \frac{B_{\min } D_{\eta, \mathrm{j}}(v)}{v \tau_{\mathrm{B}} \xi} \frac{\partial}{\partial \xi}\left[\frac{\left(1-\xi^{2}\right)}{\xi} \oint \eta \frac{d l_{\mathrm{p}}}{B_{\mathrm{p}}} \frac{\partial f_{0}}{\partial \xi}\right] \\
& +\frac{1}{v^{2}} \frac{\partial}{\partial_{v}}\left(A_{r, \mathrm{j}}(v) f_{0}\right)+\frac{1}{v^{2}} \frac{\partial}{\partial v}\left(v^{2} B_{v, \mathrm{j}}(v) \frac{\partial f_{0}}{\partial v}\right) .
\end{aligned}
$$

By using eigenfunctions $C_{n}$ for the first term in the right-hand side of eq. (10), such that

$$
\begin{aligned}
& \frac{B_{\min } D_{\eta, \mathrm{j}}(v)}{v \tau_{\mathrm{B}} \xi} \frac{\partial}{\partial \xi}\left[\frac{\left(1-\xi^{2}\right)}{\xi} \oint \eta \frac{d l_{\mathrm{p}}}{B_{\mathrm{p}}} \frac{d C_{n}}{d \xi}\right]+\lambda_{n} C_{n} \\
& =0,
\end{aligned}
$$

we expand $f_{0}$ with $C_{n}$ as $f_{0}(t, v, \psi, \xi)=\sum_{n=0}^{n \max } a_{n}(t, v, \psi)$ $C_{n}(\xi, \psi)$ in eq. (5) and then have following equations for $a_{n}:$

$$
\begin{aligned}
\frac{\partial a_{n}}{\partial t}= & \frac{1}{v^{2}} \frac{\partial}{\partial v}\left(A_{v} a_{n}\right)+\frac{1}{v^{2}} \frac{\partial}{\partial v}\left(v^{2} B_{v}(v) \frac{\partial a_{n}}{\partial v}\right) \\
& -D_{\eta} \lambda_{n} a_{n}+S_{n},
\end{aligned}
$$

where

$$
S_{n}=\frac{\int C_{n}\langle S\rangle_{\mathrm{B}} d \xi}{\int C_{n}^{2} d \xi}
$$

The eigenfunctions $C_{n}$ are odd (even) functions of $\xi$ for odd (even) $n$ [9]. Since the fast ion current is not related to $C_{n}$ for even $n$, we obtain $C_{n}$ and $\lambda_{n}$ for only odd $n$ in this study. $C_{0}$ and $\lambda_{0}$ are used to obtain $a_{0}$ which is related to the fast ion density and the heating power, but they are constant, $C_{0}=1$ and $\lambda_{0}=1 / 2$ and we do not need to calculate them. The $C_{n}$ for odd $n$ is zero for trapped particles [9], namely for $|\xi| \leq \xi_{\mathrm{t}}$. Here $\xi_{\mathrm{t}}$ is the absolute value of the boundary in $\xi$ between passing and trapped particles and is given by

$$
\xi_{\mathrm{t}}=\sqrt{1-B_{\min } / B_{\max }}
$$

where $B_{\max }$ is the maximum value of magnetic field on the magnetic surface. On the other hand, for passing particles, the bounce average can be replaced with the magnetic surface average defined by

$$
\langle X\rangle_{\mathrm{M}}=\oint \frac{X d l_{\mathrm{p}}}{B_{\mathrm{p}}} / \oint \frac{d l_{\mathrm{p}}}{B_{\mathrm{p}}} .
$$

Using eq. (15) in eq. (11), we have

$$
\begin{aligned}
& \frac{B_{\min }}{\xi\langle B / \eta\rangle_{\mathrm{M}}} \frac{d}{d \xi}\left\{\left(1-\xi^{2}\right) \frac{\langle\eta\rangle_{\mathrm{M}}}{\xi} \frac{d C_{n}}{d \xi}\right\}+\lambda_{n} C_{n} \\
& \quad=0
\end{aligned}
$$

in $\xi_{\mathrm{t}} \leq|\xi| \leq 1$, where $\langle\eta\rangle_{\mathrm{M}}$ and $\langle B / \eta\rangle_{\mathrm{M}}$ are calculated by numerical integration along the field line or the magnetic surface in general. For the magnetic surfaces with circular cross section and the large aspect ratio, they are approximated by the complete elliptic integrals [10], which are used in the RISK code [4]. The eigenfunctions $C_{n}$ for odd $n$ are obtained by assuming the following forms

$$
C_{n}=\left\{\begin{array}{cl}
\sum_{1 \leq \mathrm{odd} i \leq n} E_{n, i}\left(\xi-\xi_{\mathrm{t}}\right)^{\mathrm{i}} & \left(\xi_{\mathrm{t}}<|\xi| \leq 1\right) \\
0 & \left(|\xi| \leq \xi_{\mathrm{t}}\right)
\end{array},\right.
$$

and calculating the coefficients $E_{n, i}$ and the eigen values $\lambda_{n}$. We can calculate the terms in eq. (16) and then $C_{n}$ if the MHD equilibrium is decided.

The time evolution of $a_{n}(t, v, \psi)$ is calculated by solving eq. (12) with the finite difference method. In this study, we used the Crank-Nicolson method.

\section{NB Current Drive and Heating}

We evaluate the NB-driven current density, the heating power to bulk ions $P_{\mathrm{i}}$ and that to electron $P_{\mathrm{e}}$ from $f_{0}$.

First, we evaluate the NB-driven current density $\boldsymbol{j}^{\mathrm{NB}}$. For the MHD equilibrium calculation in the TOTAL code $\langle\boldsymbol{j} \cdot \boldsymbol{B}\rangle_{\mathrm{M}}$ is used as the physical quantity related to plasma current density $\boldsymbol{j}$. Correspondingly, we evaluate $\left\langle\boldsymbol{j}^{\mathrm{NB}} \cdot \boldsymbol{B}\right\rangle_{\mathrm{M}}$ here. To evaluate $\left\langle\boldsymbol{j}^{\mathrm{NB}} \cdot \boldsymbol{B}\right\rangle_{\mathrm{M}}$, we need to consider the fast ion current $\boldsymbol{j}^{\text {fast }}$ calculated from $f_{0}$ and the electron current caused by electrons dragged by the fast ion flow. In this study, we use the coefficient $c_{\text {elec }}$ to reflect the electron current effect given by the Mikkelsen model [11]. By using $c_{\text {elec }},\left\langle\boldsymbol{j}^{\mathrm{NB}} \cdot \boldsymbol{B}\right\rangle_{\mathrm{M}}$ is given by the following equation [5]

$$
\begin{aligned}
\left\langle\boldsymbol{j}^{\mathrm{NB}} \cdot \boldsymbol{B}\right\rangle_{\mathrm{M}} & =C_{\text {elec }}\left\langle\boldsymbol{j}^{\text {fast }} \cdot \boldsymbol{B}\right\rangle_{\mathrm{M}} \\
& =C_{\text {elec }} Z_{\mathrm{b}} e \int f_{0}(v, \xi)\langle\eta B\rangle_{\mathrm{B}} v d V, \\
d V=2 \pi v^{2} d v d \xi, &
\end{aligned}
$$


where, $e$ is the elementary charge. Note that the above expression implies that $f_{0}$ is regarded as the volume averaged distribution function, not the distribution function calculated from eq. (5). Correspondingly, we calculate the source term in the RHS of eq. (5) by volume averaging, not bounce averaging. It is considered that the errors due to this approximation are not large for the present purpose where only the passing particles are taken into account. This treatment is used in the ACCOME code and the results of NB current drive agreed with an experiment data [12].

Next, we evaluate the NB heating power. The heating power to bulk j particles $P_{\mathrm{j}}$ are given by

$$
P_{\mathrm{j}}=-\int \frac{1}{2} m_{\mathrm{b}} v^{2}\left\langle C_{\mathrm{bj}}\right\rangle_{\mathrm{B}} d V .
$$

In calculating integrals in eqs. (18) and (19), we use the trapezoid integration for $v$ and the Gauss-Legendre integration for $\xi$.

\section{Verification of the FP Solver}

To confirm the accuracy of the developed FP solver, we compared it with the OFMC code and the ACCOME code. In the analysis, we assumed a tokamak plasma which has a circular cross section with the major radius $R=8.5 \mathrm{~m}$, the minor radius $a=2.42 \mathrm{~m}$ and the aspect ratio $A=3.5$. as shown in Fig. 1. As the source term, we used a point source with the power $P_{\text {inj }}=10 \mathrm{~kW}$. The point source means a source of fast ions with a given velocity and a given pitch angle parameter $\xi$ on a given flux surface. Under the condition $v_{\text {th,i }} \ll v \ll v_{\text {th,e }}$, we used coefficients in eq. (9) for $C_{\mathrm{bj}}$. We used 500 meshes in $v$ with $v=1.33 v_{\text {inj }}$ at the upper boundary and used time step $\Delta t=10^{-3} \tau_{\mathrm{s}}$, where $v_{\text {inj }}$ is the initial velocity of the fast ions.

First, we compared the FP solver and the OFMC code for the heating power to electrons and ions $P_{\mathrm{e}}, P_{\mathrm{i}}$. The comparison was done at the five points in the density and

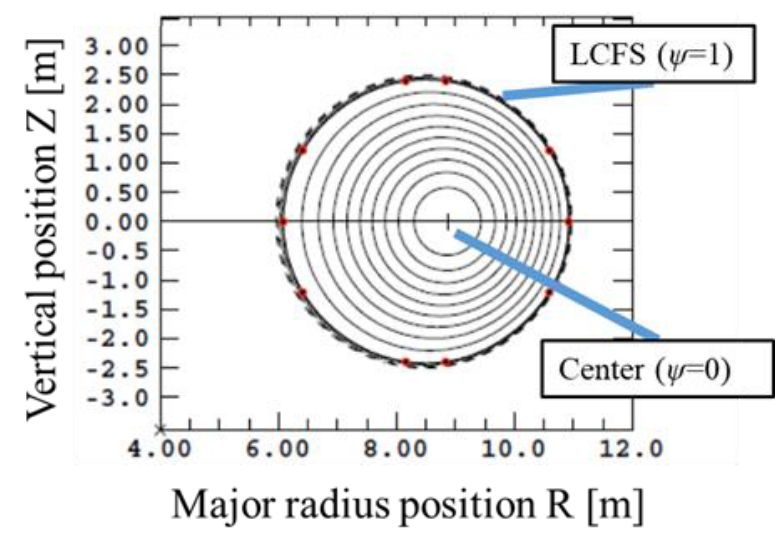

Fig. 1 Magnetic surfaces $(\psi=0,0.1, \ldots, 1)$ projected on the poloidal plane where $\psi$ means the normalized poloidal flux function. the temperature radial profiles shown in Fig. 2. These profiles were decided assuming those in future DEMO. The toroidal drift was assumed to be zero in OFMC for comparison, so that the test particle moves completely along the magnetic field. We used $E_{\mathrm{b}}=0.5,1.0,1.5 \mathrm{MeV}$ where $E_{\mathrm{b}}$ means the initial energy of fast ions. The results are shown in Fig. 3.

The results of $P_{\mathrm{e}}$ approximately matched. The OFMC code and the FP solver did not agree in $P_{\mathrm{i}}$, but $P_{\mathrm{i}}+P_{\text {th }}$ of the OFMC code and $P_{\mathrm{i}}$ of the FP solver agreed well, where $P_{\text {th }}$ is the thermalized ions power calculated by the OFMC code. This is because the fast ions are removed when its velocity reached the thermal velocity level in the OFMC code, while the fast ions are continuously deaccelerated to
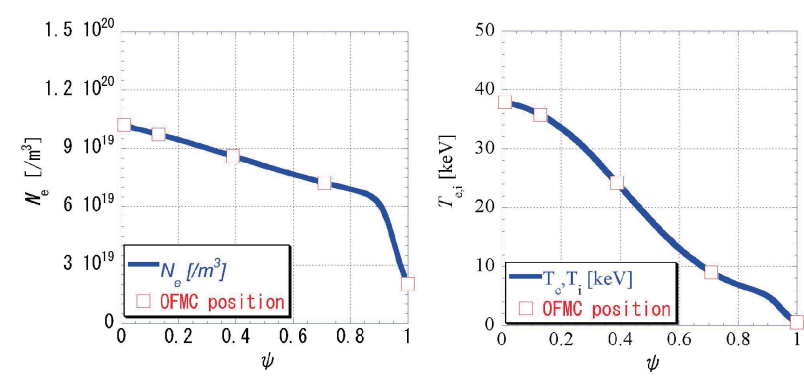

Fig. 2 Radial profile of $n_{\mathrm{e}}$ (left) and $T_{\mathrm{e}, \mathrm{i}}$ (right). Red symbols denote the positions for comparison.

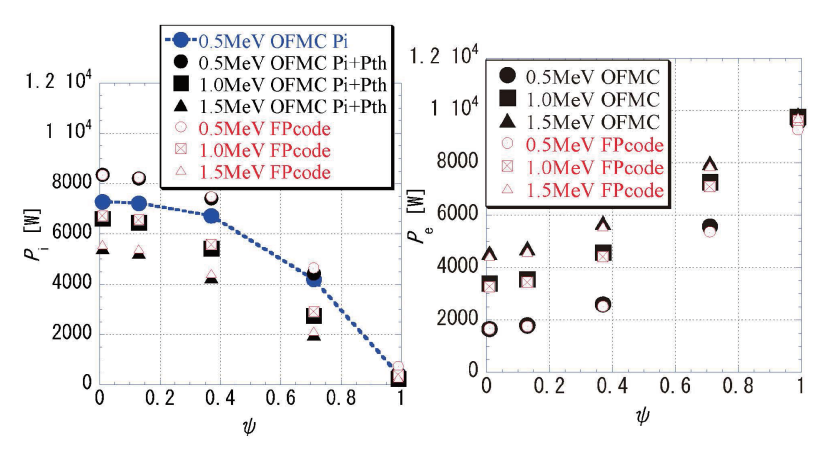

Fig. 3 Radial profiles of $P_{\mathrm{i}}$ (left) and $P_{\mathrm{e}}$ (right). $P_{\mathrm{i}}+P_{\mathrm{th}}$ means total power transferred to bulk ions from fast ions in OFMC.

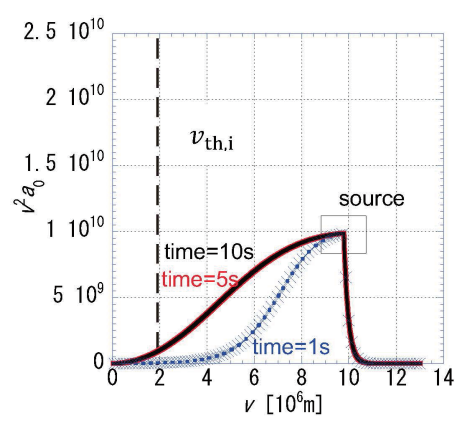

Fig. 4 Profiles of $v^{2} a_{0}$ at $\psi=0.01$. The black dashed line means $v=v_{\mathrm{th}, \mathrm{i}}$. 


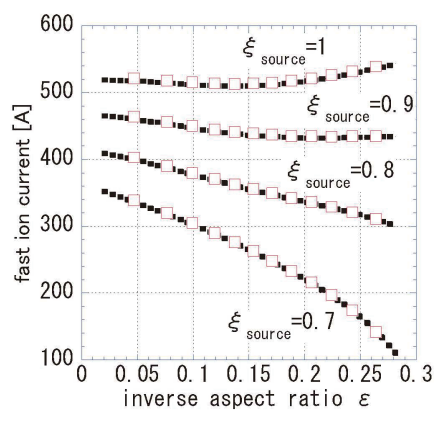

Fig. $5 \varepsilon$ dependence of $\left\langle\mathrm{I}_{\text {tor }}^{\text {fast }}\right\rangle_{\mathrm{S}}$ where $\varepsilon$ is the inverse aspect ratio; $\varepsilon=$ minor radius $/$ major radius.

$v=0$ transferring its energy to the bulk plasma ions using coefficients given by eq. (9) before they are removed in the FP solver. Figure 4 shows profiles of $v^{2} a_{0}(v)$ which is the distribution of magnitude of fast ion velocity obtained by the FP solver. The fast ions are not continuously accumulated at $v=v_{\text {th,i }}$ but the profile reaches a steady state. Thus, we have confirmed that the FP solver can provide the approximately accurate power transferred to ions and that to electrons, which are needed to solve energy transport equations for ions and electrons in the TOTAL code.

Next, the results of comparing the ACCOME code and the FP solver on the fast ion current are shown in Fig. 5 in the conditions of $n_{\mathrm{e}}=5 \times 10^{19} \mathrm{~m}^{-3}, T_{\mathrm{e}, \mathrm{i}}=5 \mathrm{keV}$. Here, $\left\langle I_{\text {tor }}^{\text {fast }}\right\rangle_{\mathrm{S}}$ means the fast ion toroidal current averaged along the contour of the flux in the poloidal plane and is described by

$$
\left\langle I_{\text {tor }}^{\text {fast }}\right\rangle_{\mathrm{S}}(\psi)=F(\psi)\left\langle j_{\|}^{\text {fast }} B\right\rangle_{\mathrm{M}},
$$

where $F(\psi)$ is decided by the magnetic surface shape. The same formula is used for $F(\psi)$ in the ACCOME code and in the FP solver. We used point sources with $\xi=1.0$, $0.9,0.8,0.7$ and energy of $E_{\mathrm{b}}=1.0 \mathrm{MeV}$. All results of the FP solver agreed well with the ACCOME code. This is because the ACCOME code also uses coefficients given by eq. (9). We found that the time needed to reach the steady state is about $2 \mathrm{~s}$ in the calculation at the magnetic axis. This value almost agreed with the estimated time $2.1 \mathrm{~s}$ from an expression, $\left(\tau_{\mathrm{s}} \ln \left(v_{\mathrm{inj}} / v_{\mathrm{c}}+1\right)\right) / 3$ given by Gaffey [13].

\section{Introduction into the TOTAL Code}

The FP solver was introduced into the TOTAL code together with a deposition code. The deposition code is a Monte Carlo code to analyze the spatial distribution of fast ions generated from injected NB through ionization or charge-exchange reactions. It was developed based on that used in the ACCOME code [5]. We get the volume averaged source term $\langle S\rangle_{\mathrm{v}}(v, \xi, \psi)$ summing up the point sources on the magnetic surface obtained by the deposition code. The stopping cross section to decide an ionization position of a test particle is given by the numerical fit by $\mathrm{S}$. Suzuki et al. [14]. By coupling the FP solver and the depo-

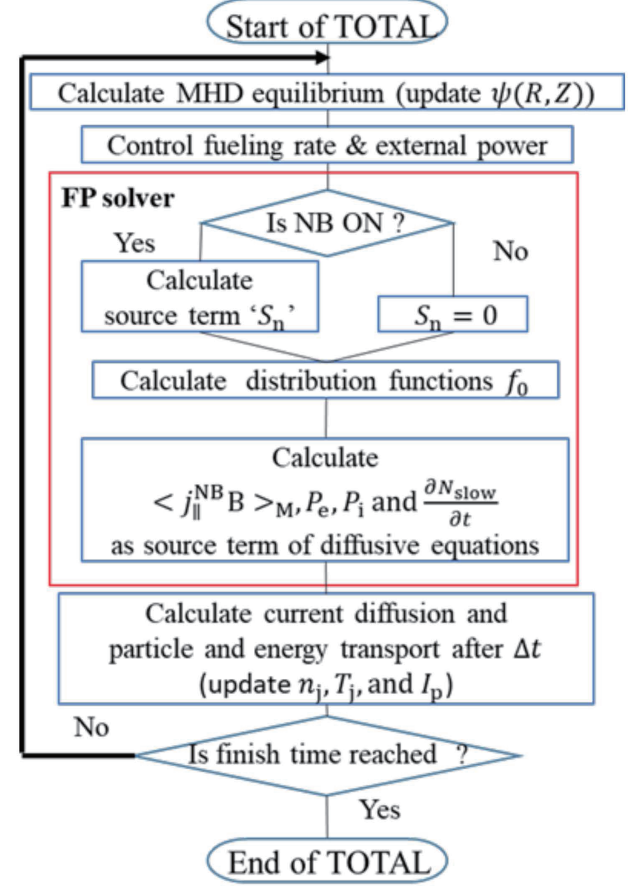

Fig. 6 Outline of TOTAL code implemented with the FP solver. $n_{\mathrm{j}}, T_{\mathrm{j}}, I_{\mathrm{p}}$ and $\Delta t$ means density, temperature of particle $\mathrm{j}$, the plasma current and the time interval.

sition code, it became able to evaluate NB heating power and driven current with the TOTAL code. We show the flow chart of the TOTAL code in Fig. 6. The TOTAL code is a 1-D particle and energy transport, 1-D current diffusion and 2-D equilibrium calculation code. The NB-driven current, the heating power and the thermalized ion number calculated by the FP solver are used as the source terms of the corresponding equations. We define the number of the thermalized ions per unit time $\partial N_{\text {slow }} / \partial t$ by subtracting the change rate of the number of fast ions from the number of particles provided by the source per unit time:

$$
\frac{\partial N_{\text {slow }}}{\partial t}=\int S d V-\frac{\partial N_{\mathrm{b}}}{\partial t},
$$

where

$$
\frac{\partial N_{\mathrm{b}}}{\partial t}=\int \frac{\partial f_{0}}{\partial t} d V
$$

We show an example of a tokamak plasma operation scenario using NB injection, which was calculated with the TOTAL code. In the example, we used pellet injection for fueling, RF injection for heating and NB injection for current drive and heating. The heating power profile by $\mathrm{RF}$ was given by a fixed function, while the RF power $P_{\mathrm{RF}}$ was adjusted to keep the fusion power $P_{\text {fus }}=1.46 \mathrm{GW}$. The pellet injection rate was adjusted to keep the line averaged electron density $\overline{n_{\mathrm{e}}}=1.2 n_{\mathrm{GW}}=8.02 \times 10^{19} \mathrm{~m}^{-3}$, where $n_{\mathrm{GW}}$ is the Greenwald density. The MHD equilibrium was determined to satisfy the major radius $8.5 \mathrm{~m}$, the minor radius $2.42 \mathrm{~m}$, and the elongation 1.65 , without an $\mathrm{X}$ 


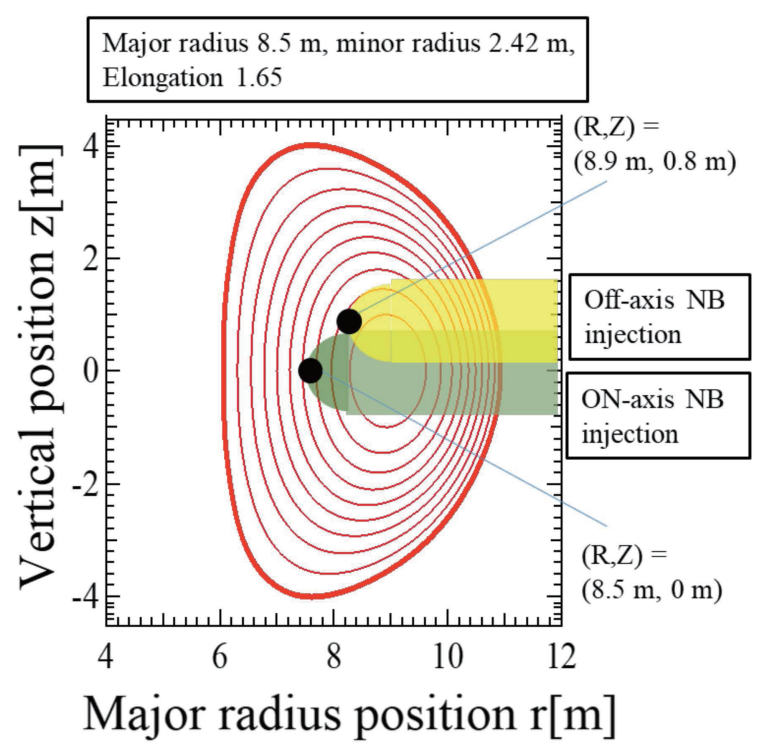

Fig. 7 Magnetic surfaces and injected NB lines projected on the poloidal plane. The red line denotes the magnetic surfaces in the TOTAL code. The green and yellow areas denote NB lines for on-axis and off-axis injection, respectively, where the height indicates the cross-sectional diameter $2 a_{\mathrm{B}}$.

point. We assumed two NB units; one is an on-axis injection and the other is an off-axis injection. The deuterium atoms with $1 \mathrm{MeV}$ are tangentially injected parallel to the equatorial plane as shown in Fig. 7. The beams had circular cross sections with the radius $a_{\mathrm{B}}=0.75 \mathrm{~m}$ and the radial profile of beam density is given by a Gaussian distribution $w$, that is,

$$
\begin{aligned}
& w\left(r_{\mathrm{B}}, \theta_{\mathrm{B}}\right)=\left\{\begin{array}{cc}
\frac{1.582}{\pi a_{\mathrm{B}}^{2}} \exp \left(-\left(\frac{r_{\mathrm{B}}}{a_{\mathrm{B}}}\right)^{2}\right) & \left(r_{\mathrm{B}}<a_{\mathrm{B}}\right), \\
0 & \left(r_{\mathrm{B}} \geq a_{\mathrm{B}}\right)
\end{array}\right. \\
& \int_{0}^{a_{\mathrm{B}}} \int_{0}^{2 \pi} w\left(r_{\mathrm{B}}, \theta_{\mathrm{B}}\right) r_{\mathrm{B}} d r_{\mathrm{B}} d \theta_{\mathrm{B}}=1,
\end{aligned}
$$

where $r_{\mathrm{B}}, \theta_{\mathrm{B}}$ are polar coordinates on the cross section. The NB axis of the on-axis unit reaches the major radius position $R=8.5 \mathrm{~m}$ and the vertical position $Z=0 \mathrm{~m}$, while the NB axis of the off-axis unit reaches $(R, Z)=(8.9 \mathrm{~m}$, $0.8 \mathrm{~m}$ ). Figure 7 shows the magnetic surfaces and injected NB lines projected on the poloidal plane at $t=3000 \mathrm{~s}$.

In the TOTAL code, diffusion of the induced current is calculated to satisfy the given value of the total plasma current. During $t=0 \mathrm{~s}$ to $3000 \mathrm{~s}$, the plasma current was kept at 12.3 MA by the induced current and the bootstrap current. At $t=3000 \mathrm{~s} \mathrm{NB}$ injection was started and continued until the end of simulation, $t=8000 \mathrm{~s}$, while the plasma current was fixed at 12.3 MA. The total NB power, sum of the on-axis NB power and the off-axis NB power, was adjusted so that the sum of the NB driven current and the bootstrap current became the total plasma current, namely the induced current became zero. The ratio of the on-axis
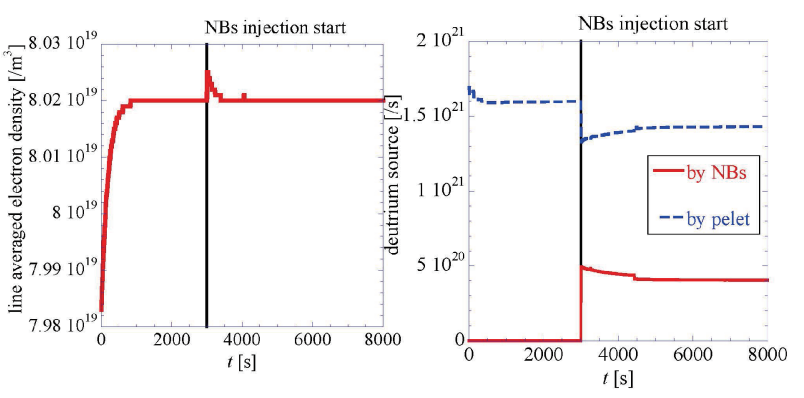

Fig. 8 Time evolution of $\overline{n_{\mathrm{e}}}$ (left) and the particle sources (right).



Fig. 9 Time evolution of $P_{\text {fus }}$ and the heating sources.

NB power and the off-axis NB power was adjusted so that the safety factor $q$ at $\rho=0.1$ became 2.4. Here $\rho$ is the magnetic surface label defined by

$$
\rho=\sqrt{\Phi / \Phi_{\text {surf }}}
$$

where $\Phi$ and $\Phi_{\text {surf }}$ are the toroidal flux enclosed by the magnetic surface and the plasma surface, respectively. Control of the safety factor near the plasma core is valid to avoid the MHD instabilities. The results are shown in Figs. 8, 9, 10 and 11 .

Figures 8 and 9 show the time evolution of $\overline{n_{\mathrm{e}}}$ and $P_{\text {fus }}$ together with the corresponding sources. $\overline{n_{\mathrm{e}}}$ and $P_{\text {fus }}$ reached the target value shortly after the start of the calculation, and then followed the target value until the end of the calculation. At $t=3000 \mathrm{~s}$, the number of particles and heating by NB injection increased and the density and the fusion power increased transiently. The pellet supply and RF heating power were then adjusted so that they returned to respective target values.

Figure 10 (a) shows the time evolution of the NB driven current, the bootstrap current, the induced current and the plasma current. As the result of adjustment of the power of NB units, the induced current quickly fol- 

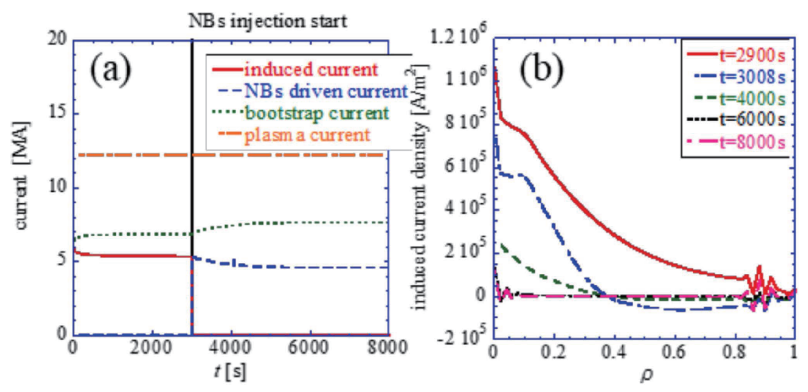

Fig. 10 (a) Time evolution of currents and (b) radial profiles of the induced current density.
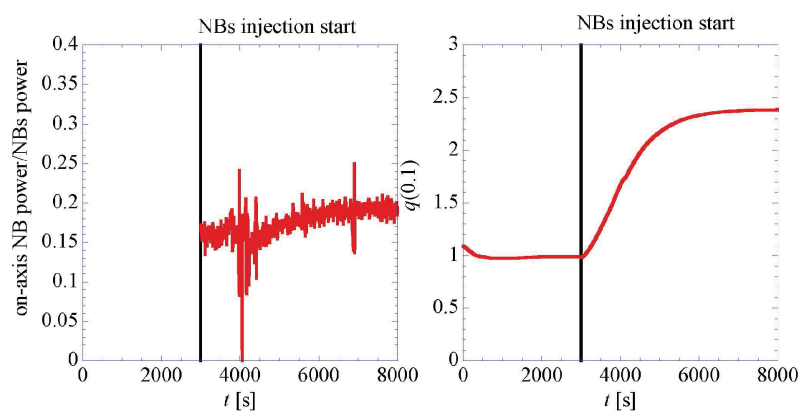

Fig. 11 Time evolution of $f_{\mathrm{ON}}$ (left) and $q(0.1)$ (right).

lowed the target value $(=0)$. However, since the rise of the NB driven current was shorter than the characteristic time of the current diffusion in the condition like DEMO, the local induced current density did not become zero even if the total induced current was controlled to be zero, as shown in Fig. 10 (b). Note that the induced current contains not only the current caused by ohmic coil but the current by time variation of the NB driven current and the bootstrap current. As a result of continuously adjusting the NB power so that the total induced current became zero, the local induced current density became zero everywhere in the plasma at $t=8000 \mathrm{~s}$. Figure 11 shows the time evolution of $f_{\mathrm{ON}}$ and $q(\rho=0.1)$. Here, $f_{\mathrm{ON}}$ means the ratio of on-axis NB power to the total NBs power. As the local induced current density became zero, $q(\rho=0.1)$ approached the target value (2.4).

From these results, it is shown that the actuator parameters necessary for controlling the $\overline{n_{\mathrm{e}}}, P_{\text {fus }}$ and current distribution can be evaluated and the operation scenario can be studied by the TOTAL code.

\section{Summary}

We developed a bounce averaged Fokker-Planck equation solver by eigenfunctions expansion and the finite different method. In the solver the time dependent distribution function for the fast ions generated by NB injection is evaluated by using eigenfunctions decided by the MHD equilibrium only and solving 1-D partial differential equations for velocity, so that the high-speed analysis is possible.

We evaluated the NB heating power and the driven current from the distribution function. In steady state, the results agreed with those obtained by the ACCOME and the OFMC codes.

The developed solver was introduced into the TOTAL code together with the NB deposition code used in ACCOME. We simulated a DEMO operation scenario using NB injection with the TOTAL code. From the results, it was confirmed that DEMO operating scenarios can be examined and the NB parameters needed in the DEMO operation can be evaluated with the improved TOTAL code.

There are many effects not included in the developed solver; for example, the finite orbit effect and the exact thermalization loss effect. In future, we will add simple algorithm to include these effects with high-speed analysis. In addition, we will search best actuator parameters to achieve full non-inductive current drive and rating fusion power by several TOTAL code simulations.

\section{Acknowledgment}

This work was carried out as a part of the Tokamak plasma collaboration Research Program in QST, and partly supported by JSPS KAKENHI JP17H06231.

[1] K. Yamazaki and T. Amano, Nucl. Fusion 32, 633 (1992).

[2] M. Toma et al., Plasma Phys. Control. Fusion 57, 095007 (2015).

[3] N. Hayashi et al., 42nd EPS Conf. on Plasma Physics (Lisbon, Portugal, 22-26 June 2015) P5.145 (http://ocs.ciemat.es/EPS2015PAP/pdf/P5.145.pdf).

[4] M. Schneider et al., Nucl. Fusion 55, 013003 (2015).

[5] K. Tani, M. Azumi and R.S. Devto, J. Comput. Phys. 98, 332 (1992).

[6] K. Tani et al., J. Phys. Soc. Jpn. 50, 1726 (1981).

[7] J. Killen et al., Computational Methods for Kinetic Models of Magnetically Confined Plasmas (New York, Springer, 1986).

[8] F.F. Karney, Computer Physics Reports 4, 183 (1986).

[9] J.G. Cordey, Nucl. Fusion 16, 499 (1976).

[10] B. Wolle et al., J. Plasma Phys. 57, 793 (1997).

[11] D.R. Mikkelsen and C.E. Singer, Nucl. Technol./Fusion 4, 237 (1983).

[12] T. Oikawa et al., Nucl. Fusion 40, 435 (2000).

[13] J.D. Gaffey, Jr., J. Plasma Phys. 16, 149 (1976).

[14] S. Suzuki et al., Plasma Phys. Control. Fusion 40, 2097 (1998). 